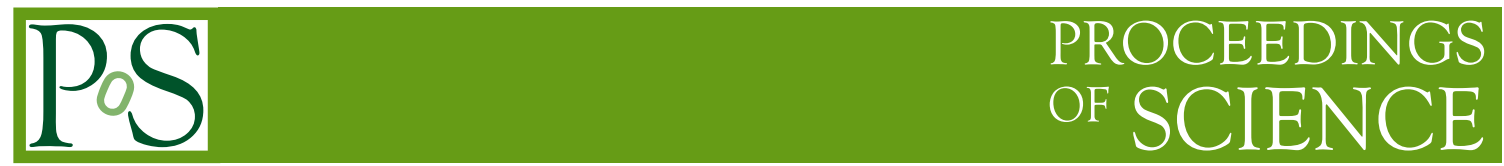

\title{
High density matter physics at J-PARC-HI
}

\section{Takao Sakaguchi*, for the J-PARC-HI collaboration}

Brookhaven National Laboratory, Physics Department

E-mail: takao@bnl.gov

Physics prospective of the high density matter using heavy ions collisions is presented. The JPARC-HI project which is a unique lab to tackle the high density matter physics is described. The world highest rate of heavy ion beam of $10^{11} \mathrm{~Hz}$ is aimed at J-PARC-HI which enables us to perform measurements of hadrons, fluctuation of conserved quantities, dileptons, multi-strange hypernuclei. New event selections are also discussed to reach a highest baryon density on the ground.

Corfu Summer Institute 2018 "School and Workshops on Elementary Particle Physics and Gravity" (CORFU2018)

31 August - 28 September, 2018

Corfu, Greece

${ }^{*}$ Speaker. 


\section{Introduction}

Decades of the QCD matter study at high temperatures became fruition at RHIC and LHC energies, where a new phase of the QCD matter, quark gluon plasma (QGP), was discovered and has been studied in detail. However, the QCD has rich phase structure not only in the high temperature side but also in the high baryon density side, about which we know very little. Figure 1 shows the QCD structure known to date. The high baryon density side supposedly contains the critical

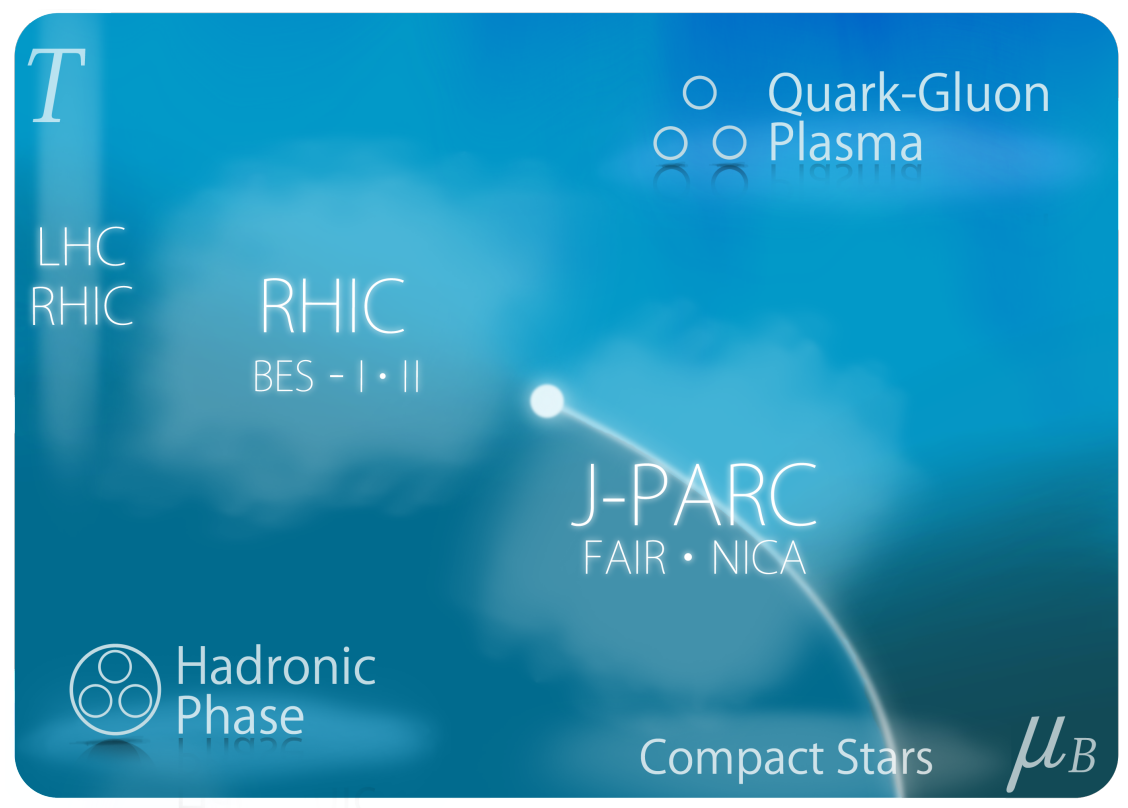

Figure 1: QCD phase structure with the $T-\mu_{b}$ regions that existing and proposed experiments are covering.

point where the cross-over phase boundary turns into the first order phase transition. As going to even higher density, the matter phase called quarkyonic or color superconductivity are expected to manifest. This high density region is important not only for the nuclear physics, but also for astrophysics, since these matters may be produced by neutron-star mergers and/or at the core of neutron-stars. For studying the properties of such a high density matter, several future projects including FAIR [1], NICA [2], and SPS have been proposed to accelerate and collide heavy ions. The J-PARC is also planning to accelerate heavy ions at the similar energies, but at a higher beam intensity, after a modest upgrade to the already operating facility. We will present perspective of physics outcome at J-PARC with the heavy ion beams as well as the status of the project. This new project is called as the J-PARC-HI project.

\section{Heavy ion acceleration at J-PARC}

Figure 2 shows a site view of the J-PARC facility with the planned addition for accelerating heavy ions. The protons from the ion source are accelerated to $400 \mathrm{MeV}$ by the LINAC, and injected to the $3 \mathrm{GeV}$ RCS (Rapid Cycle Syncrotron). The MR (Main Ring) receives protons from the RCS and accelerates them up to $50 \mathrm{GeV}$ (currently, it is operated up to $30 \mathrm{GeV}$ ). For the 


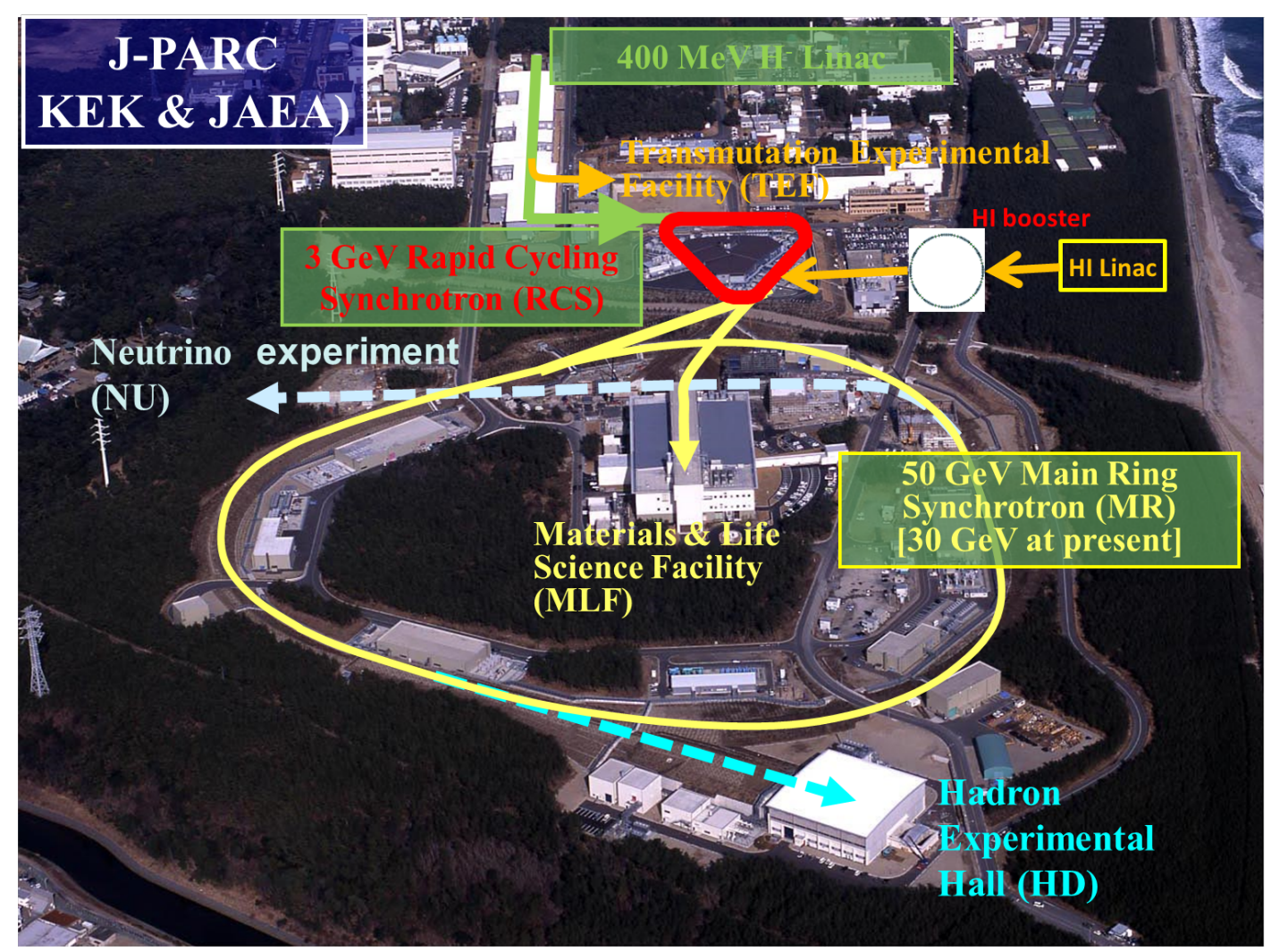

Figure 2: Site view of the J-PARC facility with the illustration of the current configuration and the proposed upgrade for heavy ion acceleration.

machine time for the material and life science facility (MLF), the protons are provided directly from the RCS. When accelerating heavy ions, one needs to construct a new ion source followed by a new LINAC and a booster for injecting heavy ions to the RCS, since the existing LINAC can only accept protons due to its rigidity constraint. This acceleration scheme is depicted in Fig. 3 in more detail. By tuning the operation parameters, this scheme can simultaneously accelerate and provide protons and heavy ions, if the protons are to be provided to the MLF and the heavy ions are to the hadron experimental hall. This will allocate decent amount of machine time for heavy ion experiments since the current machine time is dominated by the experiments at the MLF. The upgraded J-PARC, J-PARC-HI, will be flexible enough to provide ions from protons up to Uranium at the lab energy of $E=1-50 \mathrm{GeV} / \mathrm{n}$. In case of Uranium, the incident energy will become $1-19 \mathrm{GeV} / \mathrm{n}$ which corresponds to $\sqrt{s_{N N}}=1.9-6.2 \mathrm{GeV}$. The beam rate will be as high as $10^{11} \mathrm{~Hz}$ which is about one order higher than the anticipated rate at FAIR/CBM. The comparison of the performance with other facilities is shown in Fig. 4. With $0.1 \% \lambda_{I}$ target, one can expect the event rate as high as $100 \mathrm{MHz}$. This event rate will provide the one-year-run statistics at AGS at BNL within five minutes of running at J-PARC-HI. The yields of $\rho, \omega$, and $\phi$ decaying into electrons are expected to be $10^{10}$ to $10^{12}$, that of hypernuclei will be $10^{4}$ to $10^{12}$ [3], and that of strangelets will be 1 to $10^{2}$ [4], with a one month of machine time at this event rate, without consideration of duty factors. 


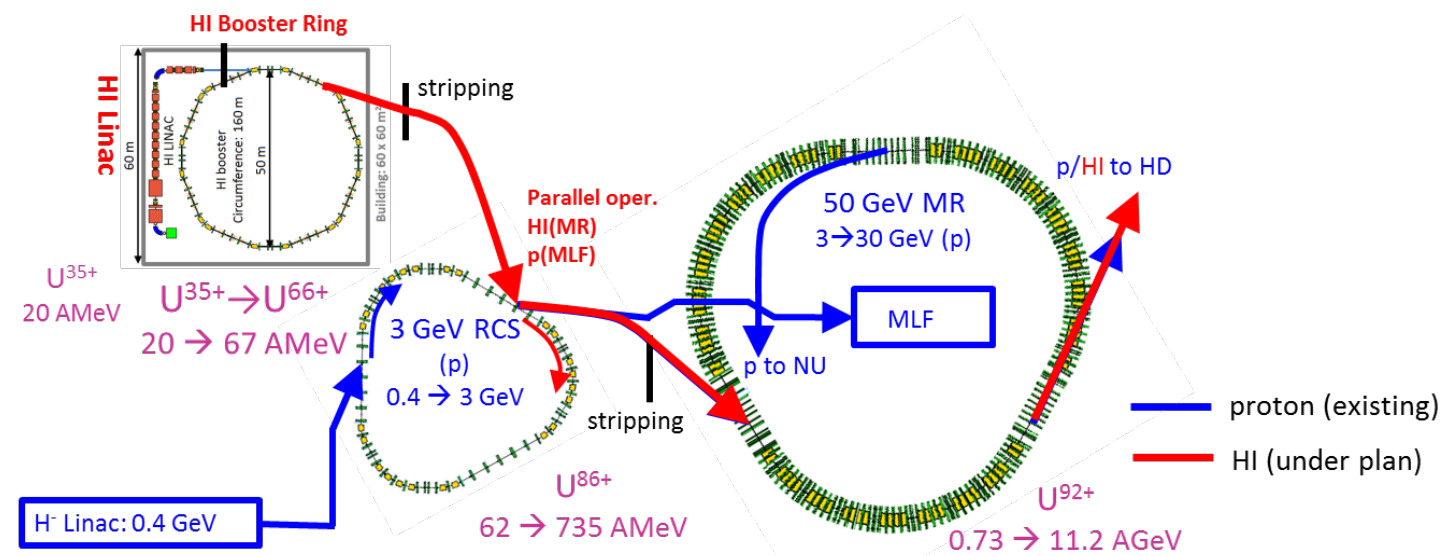

Figure 3: Schematic presentation of the beam acceleration and transport scheme for the J-PARC-HI. During the time that protons are transported to the MLF (material and life science experimental facility), heavy ions can be accelerated and transported to the hadron experimental hall through the MR.

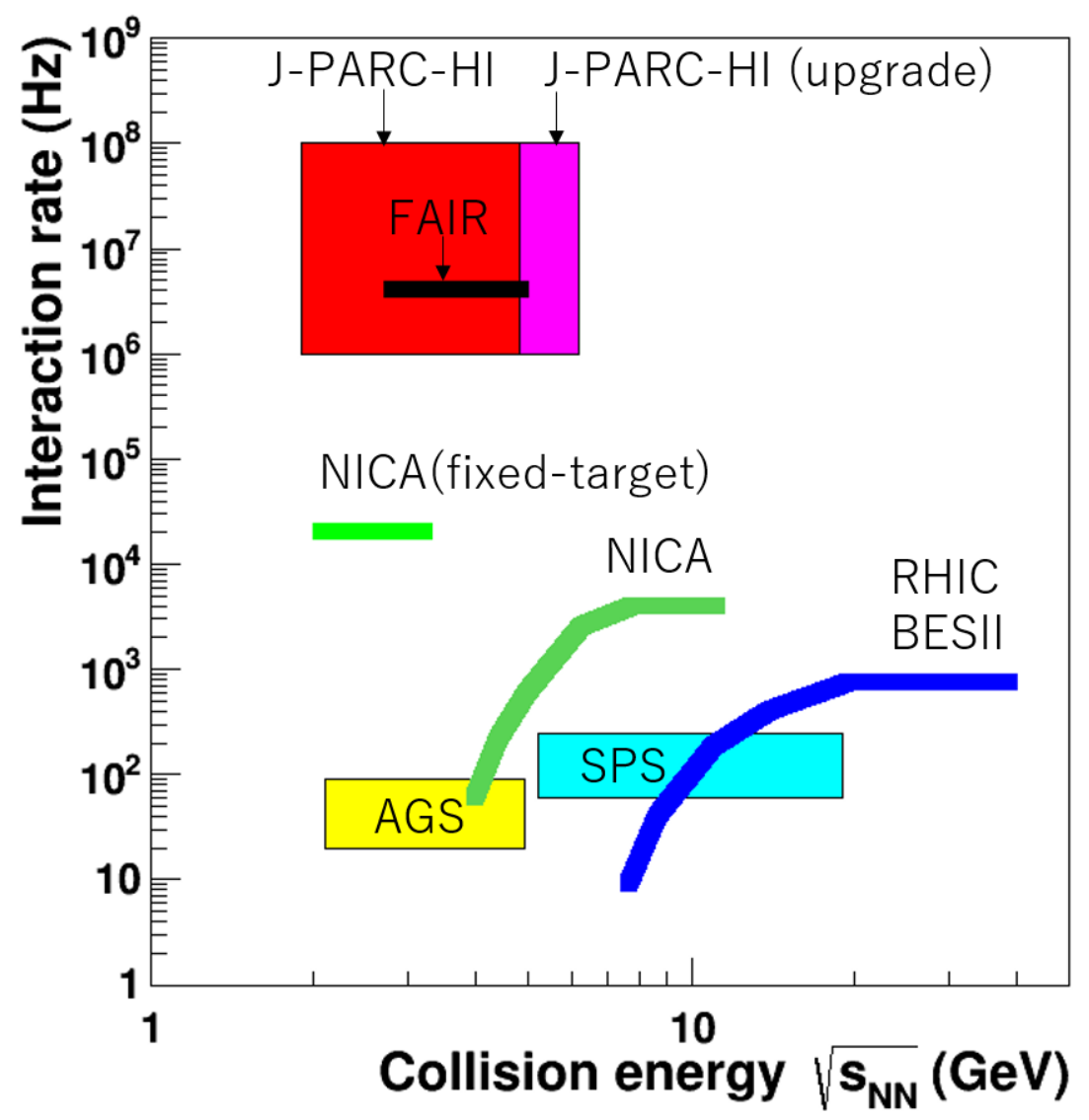

Figure 4: Interaction rates and collision energies available at the various accelerator facilities in the world. FAIR and NICA (fixed target) provides similar beam energies as J-PARC-HI. The anticipated interaction rate can be higher for J-PARC-HI. 


\section{Physics perspective}

\subsection{Collision dynamics at J-PARC-HI energy}

At the J-PARC energy, which is similar to the AGS energy, one expects to observe a high density matter created in the midrapidity region. The left side of Fig. 5 shows the $d N / d y$ of netprotons [5]. As going to lower energies from RHIC, SPS to AGS, the distribution tends to concen-
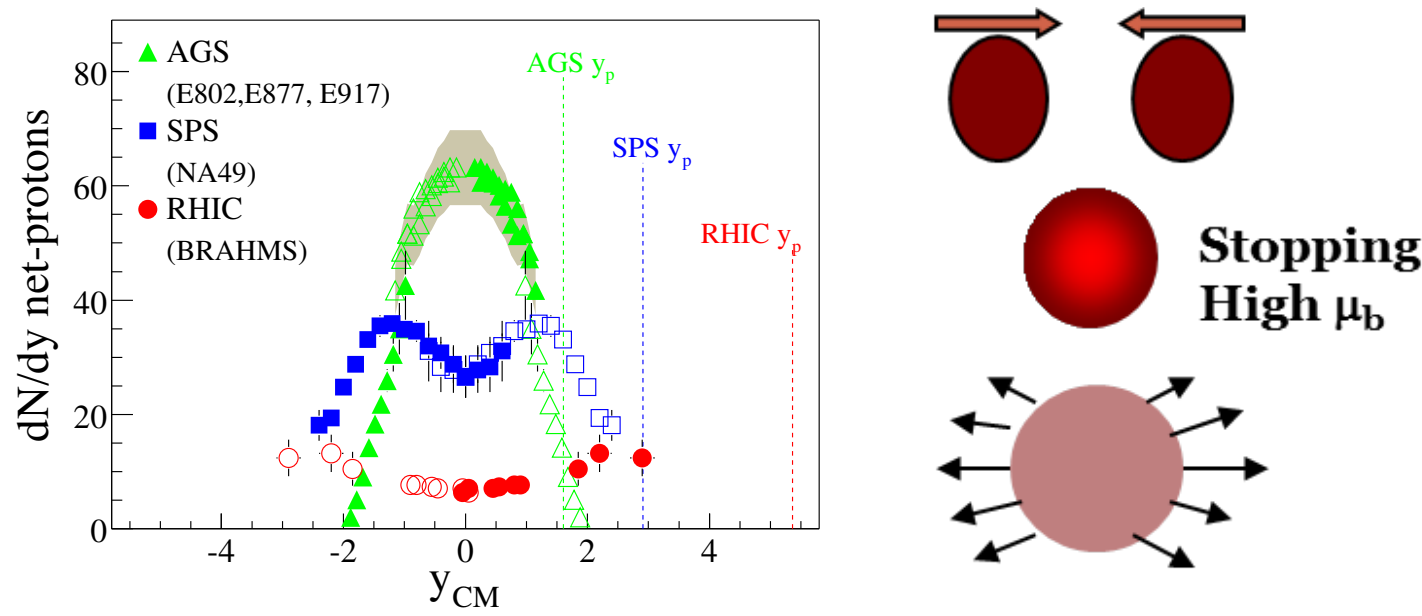

Figure 5: (left) Net-proton $d N / d y$ as a function of rapidity for AGS, SPS and RHIC energies. (right) Schematic drawing of the collision dynamics at AGS (baryon stopping).

trate around $y_{C M}=0$. At RHIC, the incoming ions are so fast that they pass through each other, therefore net-protons at the mid-rapidity is low. At the AGS, the ions are stopped by the friction with other ions and therefore the net-protons becomes large, meaning a high baryon density matter is created. This phenomena is called as baryon stopping and depicted in the right side of the Fig. 5 . From a hadron cascade calculation, JAM [6], the maximum baryon density for Au+Au collisions at this energy is estimated as $\sim 6 \rho_{0}$ and that for $\mathrm{U}+\mathrm{U}$ collisions as $\sim 8.6 \rho_{0}$.

Figure 6 shows the particle ratios as a function of $\sqrt{s_{N N}}$ [7]. The nominators are numbers of protons or the particles including open strangeness, while the denominators are the numbers of $\pi^{+/-}$which represent the total particle multiplicities of the events. It is seen that the ratios whose nominators are strangeness-containing particles have peaks around $\sqrt{s_{N N}}=5-8 \mathrm{GeV}$. The $K^{-} / \pi$ ratios are an exception, since the $K^{-}$will dominantly be created from excitation of nucleons. These experimental results show that the strangeness fraction to the total particles is largest at the J-PARC energy, suggesting a strangeness-rich matter can be created.

In order to observe a new phenomena at this energy, one has to invent a new event selection scheme. Traditionally, the heavy ion physicist chose most violent and less by using the variables called centrality. The centrality can be associated with impact parameters using a Glauber Monte Carlo calculation. However, it doesn't guarantee the selection of very high baryon density events. Here we demonstrate a new event selection from the study using the JAM code. Figure 7 show the sum of the transverse momentum of all the particles produced in an event plotted against the maximum baryon density in the event. We can see a correlation between two quantities. The right 

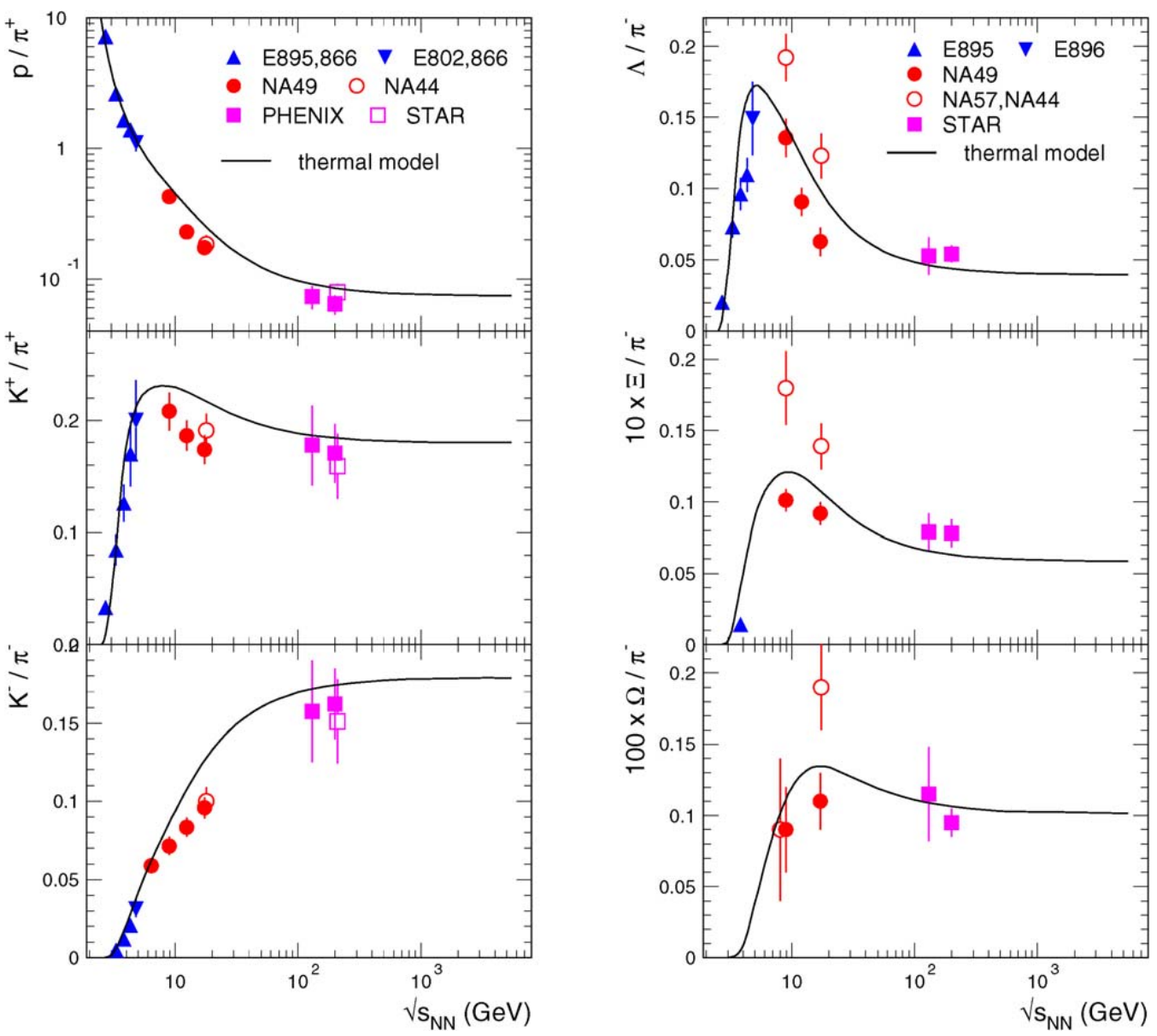

Figure 6: Compilation of particle ratios as a function of collision energies. Enhancement of the strangeness particle fraction can be seen in the yield increases of $K^{+}, \Lambda, \Xi$, and $\Omega$ which have $\bar{s}$, with respect to $\pi$ that represents the total particle multiplicity.

side of the Fig 7 shows the distribution of $\rho / \rho_{0}$, where the $\rho_{0}$ is the normal nuclear density. It is shown that a one order higher statistics can push the reach of $\rho / \rho_{0}$ higher by $1 \sigma$.

This new experimental project stimulated the theory community as well. The matter created in high energy collisions can be described by a hydrodynamical model rather well, while the one in low energy collisions can mainly described by a hadron cascade model as depicted in the left side of the Figure 8. The J-PARC-HI energy sits in transient of two pictures, therefore a model unifying the two pictures is desired. Recently, the Japanese theoretical community that has been working on heavy ion physics and is interested in the J-PARC-HI project invented a new such model [8]. The model introduced a threshold parameter $e$ which corresponds to the fluid energy density. For the region whose density is above $e$, the hadrons are turned into a parton fluid and plugged into a hydrodynamical framework. The region where the density is below $e$, the parton fluid is "cooled down" to hadrons. The right side of the Fig 8 shows the $K^{+} / \pi^{+}$and $K^{-} / \pi^{-}$as a function of $\sqrt{s_{N N}}$ compared with the calculations from the conventional JAM hadron cascade code as well as the newly proposed unified framework with two $e$ values. It is clearly seen that the unified models give better description of the data [8]. 

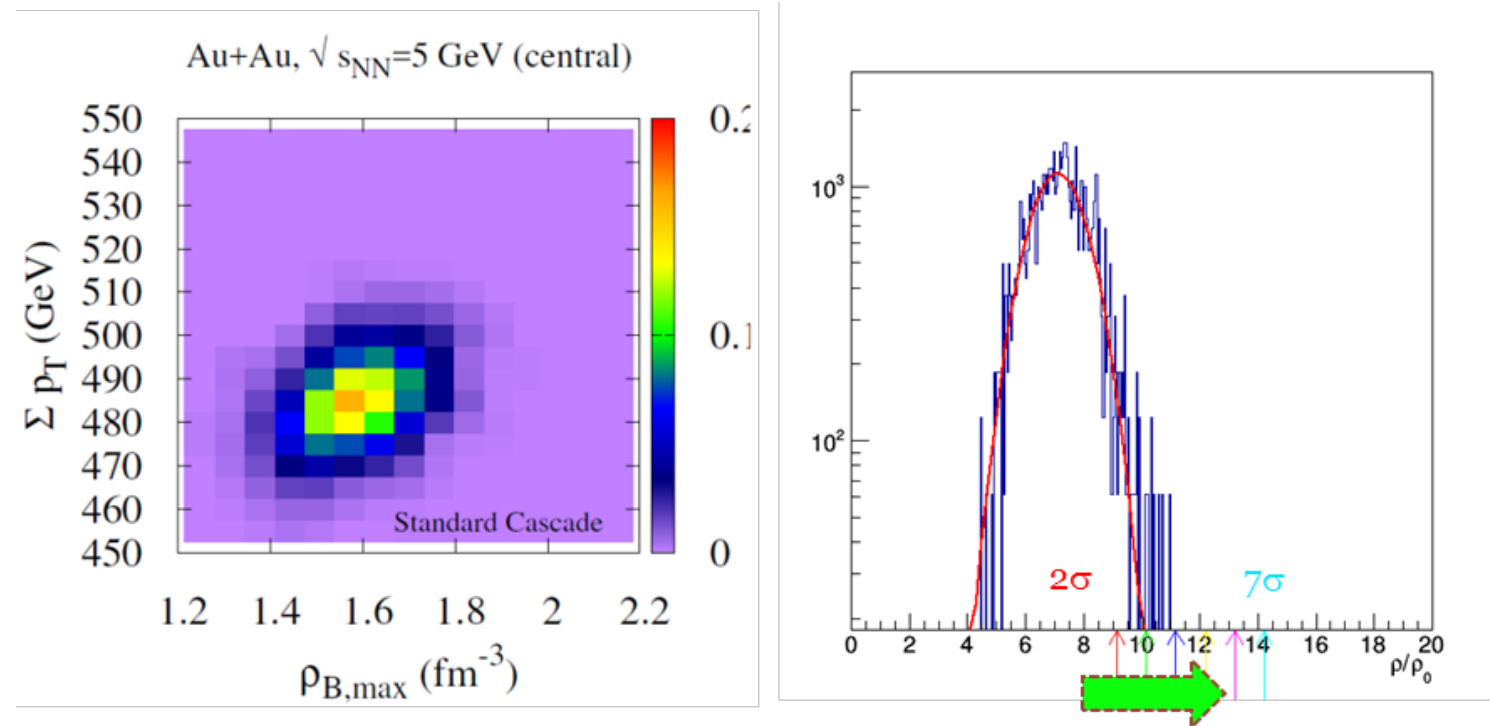

Figure 7: (left) Correlation between the sum of transverse momentum of all the particles and the maximum baryon density, $\rho_{B, \max }$, for given events, obtained by a standard hadron cascade simulation, JAM. (right) $\rho / \rho_{0}$ distribution from the same simulation. One order higher statistics will push the reach of $\rho / \rho_{0}$ higher by $1 \sigma$.
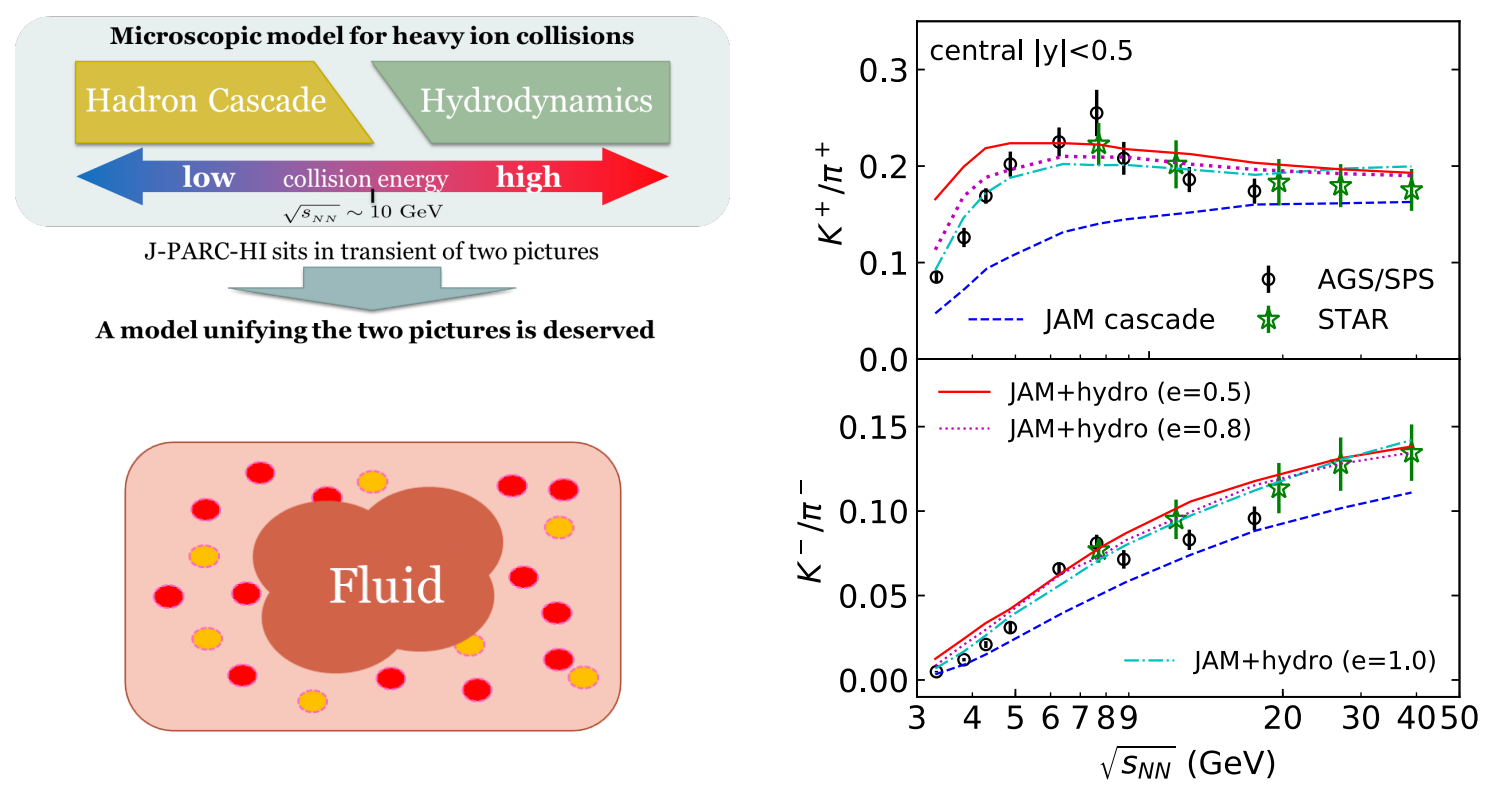

Figure 8: A theoretical effort to understand the collision dynamics at the J-PARC-HI energy. The dynamics should be described in a mixed framework of hadron cascade and hydrodynamics. The right plots show the particle ratios from the conventional hadron cascade framework and the newly proposed mixed framework. 


\subsection{Observables: Dileptons}

Dileptons are one of the important observables that have not been measured at the AGS/JPARC-HI energies. Figure 9 shows the schematic plot showing the various contribution decaying into lepton-pairs [9]. The hadrons contributing to the low mass region $\left(0.3<m_{l l}<1 \mathrm{GeV}: \rho, \omega\right.$, and

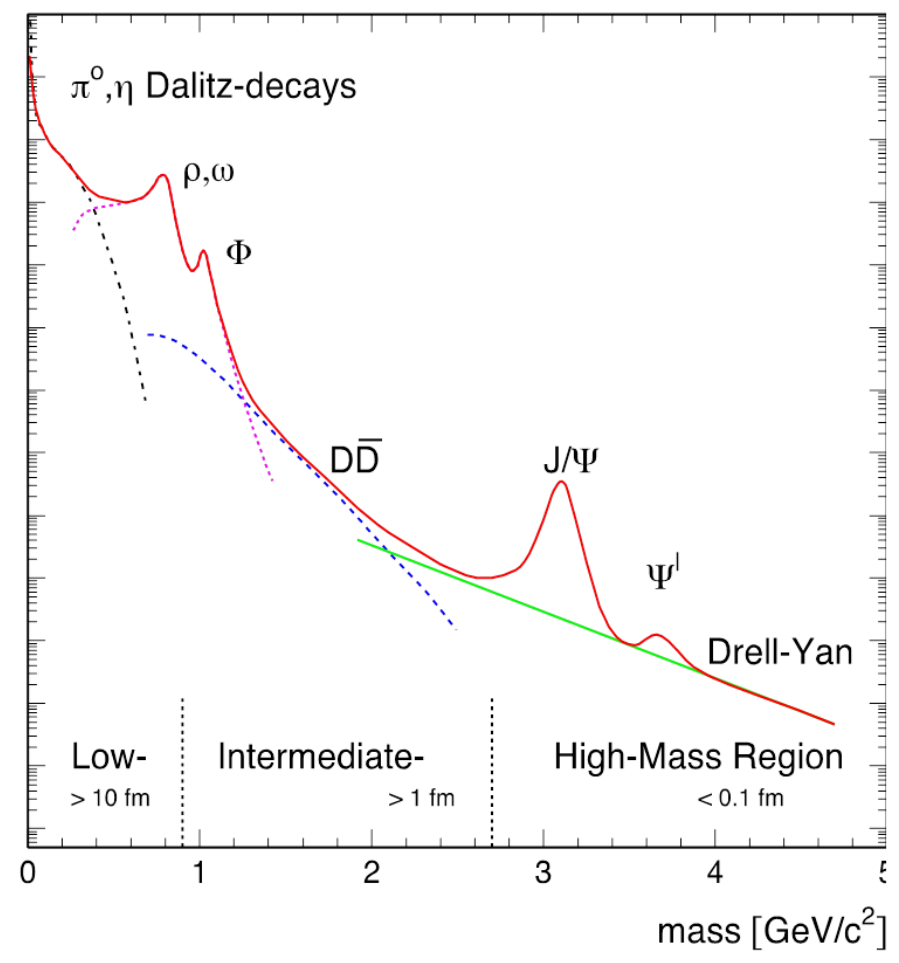

Figure 9: Schematic drawing of the lepton-pair (electron-positron pair in this case) mass distribution from various contributions.

$\phi$ ) are of course of interest for long time as they are promising observables to probe chiral symmetry restoration effect. A recent study claims that the moments of the dilepton mass spectrum:

$$
\int d m_{l l} N\left(m_{l l}\right) m_{l l}^{n} \quad(n=1,2, \ldots)
$$

can be related to the quark/gluon condensate of the system [10]. Since the uncertainty will become larger as going to a lower mass region due to a large background, a high statistics experiment such as J-PARC-HI is suitable to tackle this measurement. The mass region lower than $m_{l l}<100 \mathrm{MeV}$ is told to be the window associated with the soft mode that arises in the transition to the colorsuperconductivity phase [11]. The one above $\phi$ is dominated by leptons decaying from $c \bar{c}$ at the energy of SPS and above, where the charm production is copious. However, the $c \bar{c}$ production is highly suppressed at J-PARC energy because of kinematical limit. Therefore, this mass region is ideal for observing the thermal radiation from the matter at this energy [12].

\subsection{Observables: fluctuation}

The fluctuation of conserved quantities has been of interest since it is sensitive to the transition of the phases, especially, the critical point. One of the typical observables is the net-baryon fluctua- 
tion. In reality, one can only measure the net-protons due to difficulty of measuring neutrons. It is, however, one of the goals at J-PARC-HI to measure the neutrons and thus the genuine net-baryon fluctuations. Another interesting observable is the higher order cumulants which are more sensitive to the phase transition. Both RHIC and LHC experiments have measured up to fourth order moments (kurtosis), but the sixth and eighth moments are said to even more sensitive to the chiral phase transition as shown in Fig. 10 [13]. It is shown that the moments vary significantly around
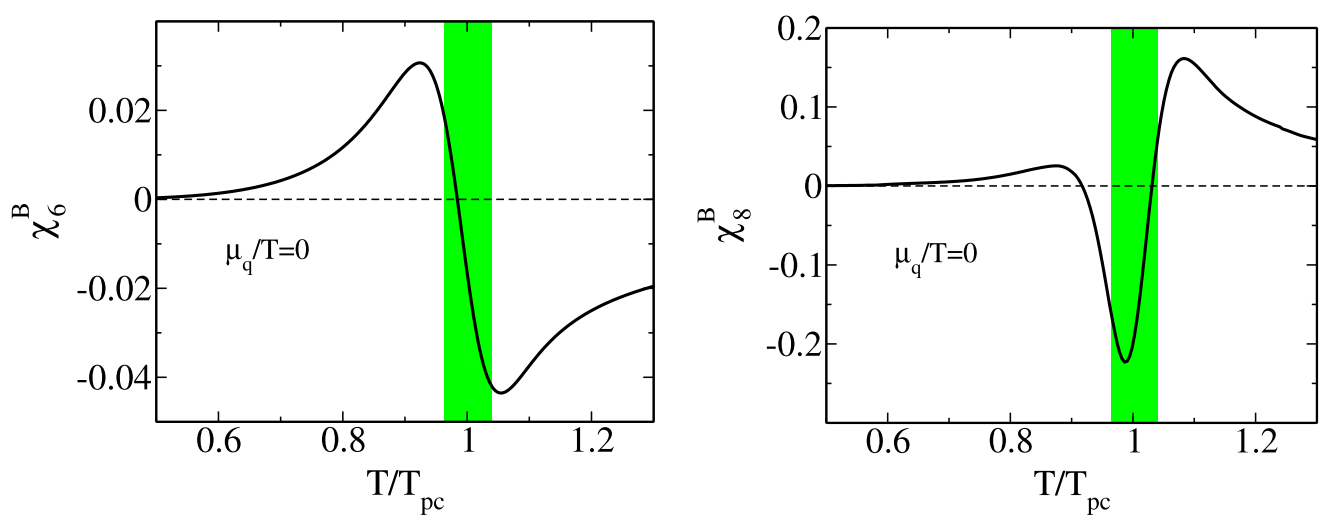

Figure 10: Sixth (left) and eighth (right) moments of the net-baryon fluctuation around the phase transition temperature $\left(T_{p c}\right)$. It is clearly seen that the moments vary significantly around $T_{p c}$.

$T_{p c}$. It should be noted that around one order magnitude higher statistics is necessary to observe moments of one order higher. Therefore, a very high statistics experiment such as J-PARC-HI is ideal to measure such an observable.

\subsection{Observables: flow}

The particle flow is sensitive to the equation of state (EOS) of the matter created in the early stages of the collisions. Depending on the order of the phase transition, the softening process will become different which results in a different flow strength. One of the flow components that are said to be sensitive is the directed flow $\left(v_{1}\right)$ and its slope as a function of rapidity $\left(d v_{1} / d y\right)$ around the mid-rapidity $(y=0)$. When softening of the EOS occurs, the nucleons in the participant region are attracted (shown in the left side of Fig. 11) and therefore $d v_{1} / d y$ becomes negative for nucleons. This is demonstrated by calculations of $d v_{1} / d y$ for protons using JAM for the case of no phase transition, first phase phase transition, and cross-over phase transition as shown in the right side of Fig. 11 [14]. It is clearly seen that in the cases of no phase transition or cross-over phase transition, the $d v_{1} / d y$ stays positive over the energies, while in the case of first order phase transition, the sign changes at the energy where the transition is expected to occur. In addition to the directed flow, high order flows are interesting in the similar sense of measuring the higher moments in the fluctuation measurement of the conserved quantities. It is also true that going to an order higher flow requires an order higher statistics where J-PARC-HI can contribute significantly.

\subsection{Observables: hypernuclei and others}

The exploration of the multi-strange hypernuclei $(S \geq 3)$ will become possible at the beam rapidity; the hypernuclei will be looked for in the beam fragment. The lifetime and the magnetic 

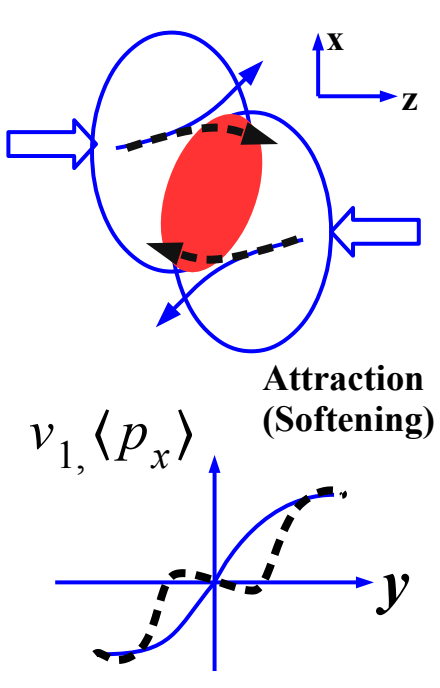

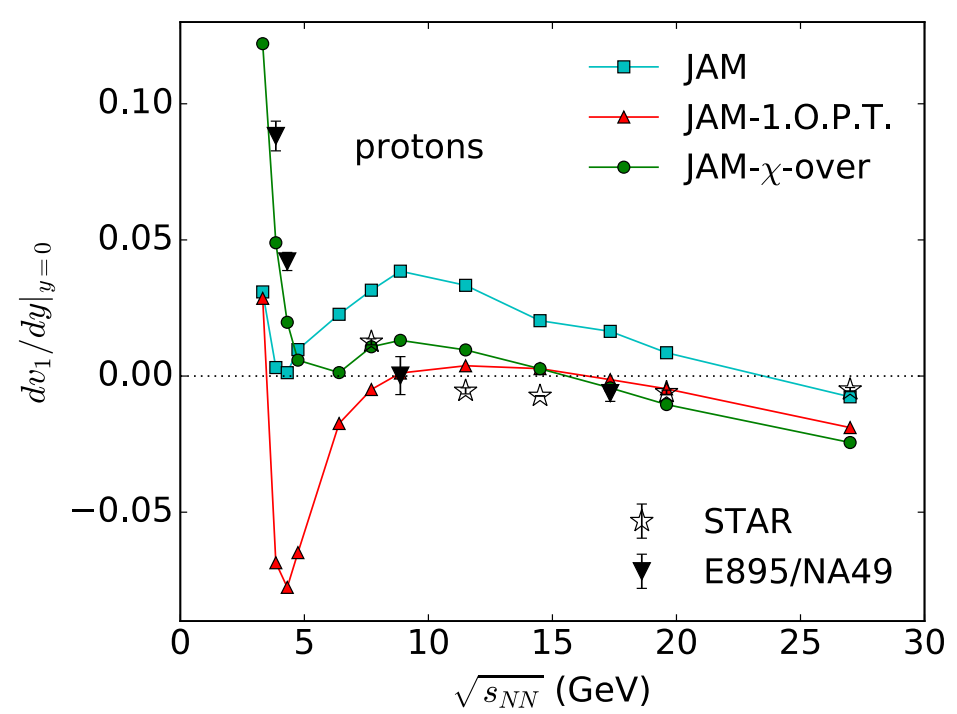

Figure 11: (left) Schematic drawing of the possible effect of EOS softening to the directed flow ( $v_{1}$, $d v_{1} / d y$ ). (right) Directed flow slopes as a function of $\sqrt{s_{N N}}$ together with hadron cascade calculations, JAM, with no phase transition, first order transition, and cross-over transition.

moment are of interest of the hypernuclei physics community since they provide information of the structure of the hypernuclei $[15,16]$. The reaction cross-section should also be measured to obtain an idea on the size of the hypernuclei.

The hadron-hadron correlations can serve to many interesting topics of the hadron physics. For instance, the $\Lambda-\Lambda$ correlation has been looked at from the point of view of dibaryon search [17], since the bound state can be detected by the strength of this correlation. The correlation can also be used to explore changes of the baryon-baryon interaction in the high baryon density environment. A recent Lattice calculation found that the $\Omega-\Omega$ creates a bound state [18]. Therefore, this correlation can be utilized in the same sense.

More exotics can be searched with very high statistics at J-PARC. Both Kaonic nucleus and strangelets have been of interest of hadron physics, since they are new forms of the hadrons whose natures are barely known. Exploring multi-quark states such as 6, 8, and/or 10 quark states will shed light on the nature of the strong interaction as well as the transition to a mini-QGP state.

\section{Proposed detectors}

The detector has to be capable of handling hits at very high rate, especially for the particle tracking part. At same time, a large acceptance will be needed for high statistics inclusive measurement of the event-by-event fluctuation. Our plan is to build and reconfigure detectors in three stages, preceded by a day-1 precursor experiment that will be described later. We will employ a high speed continuous data taking system combined with an online and a semi-online trigger system at all stages, in order to reduce the amount of data to be recorded on disk and thus to reduce the time to extract and analyze interesting events. 
The first stage focuses on the observables that can be measured at lower intensity $\left(10^{6} \mathrm{~Hz}\right)$. This is basically the soft particle production, and its correlation/fluctuation. The Fig. 12 shows a dipole hadron spectrometer to be operated at the first stage of the experiment. The main tracking

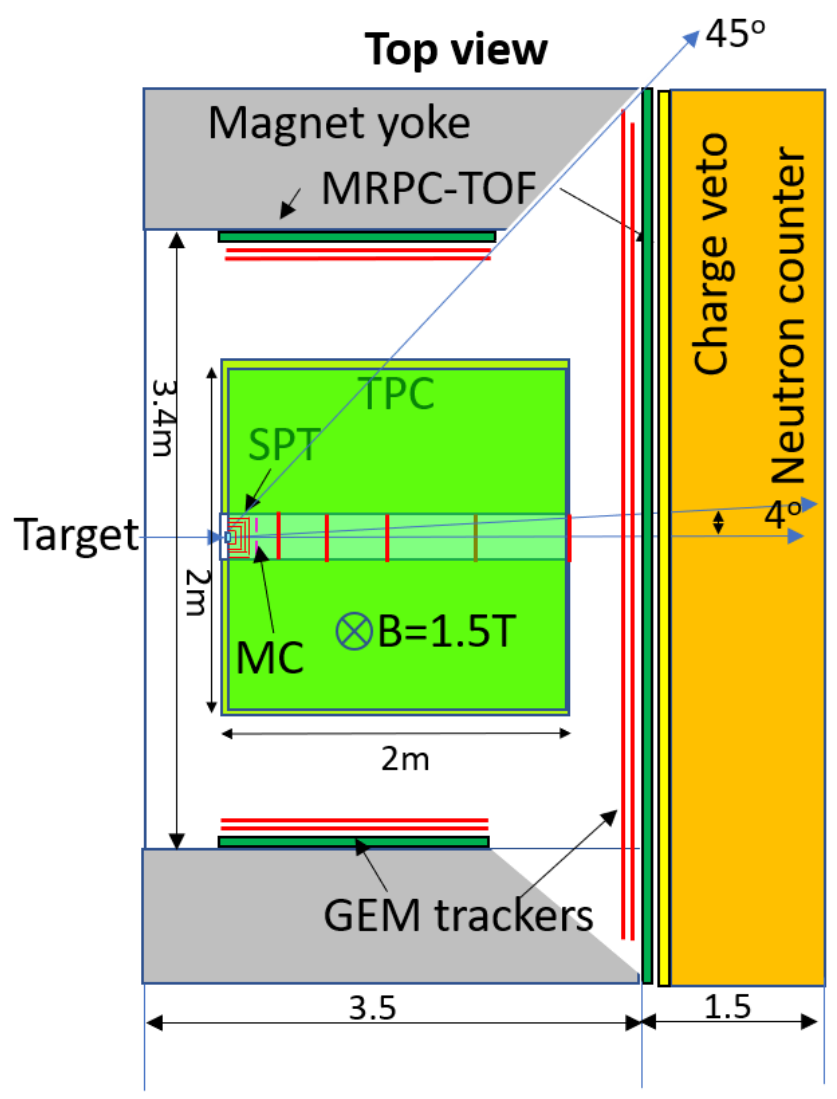

ZCAL

Figure 12: First stage experiment setup focusing on the haronic observables (hadron spectra, flow, and fluctuation).

device consists of silicon pixel detectors $\left(\theta<40^{\circ}\right)$ and a time projection chamber (TPC, $\theta>40^{\circ}$ ) which have an exclusive zenith coverage each but have a full-azimuth coverage. The tracking device is then followed by a time-of-flight (TOF) detector with multi-resistive proportional chamber (MRPC) and a neutron counter. The centrality will be determined by multiplicity counters located in front of the silicon pixel detectors and a zero-degree hadronic calorimeter located in the downstream of the beam.

The second stage experiment will perform dilepton measurement via $\mu \mu$ channel with a spectrometer shown in the Fig. 13. The TPC will be replaced by a dimuon tracker consisting of GEM trackers sandwiched with $\mathrm{Pb}$ absorbers. In order to improve the momentum and track resolution, 7layers of forward and barrel silicon pixel detectors will also be installed. We will be able to operate the detector at the rate up to $10^{7} \mathrm{~Hz}$. We have generated simulated event of hadrons decaying into muons by a modified EXODUS event generator [19] originally developed by the PHENIX experiment as shown in the left side of the Fig. 14. We then embedded the dimuons into the JAM events, processed them through a GEANT4 detector simulation software [20]. The reconstructed dimuon mass spectra which is shown in the right side of the Fig. 14 . The $\pi$ and $K$ hadrons generated by the 


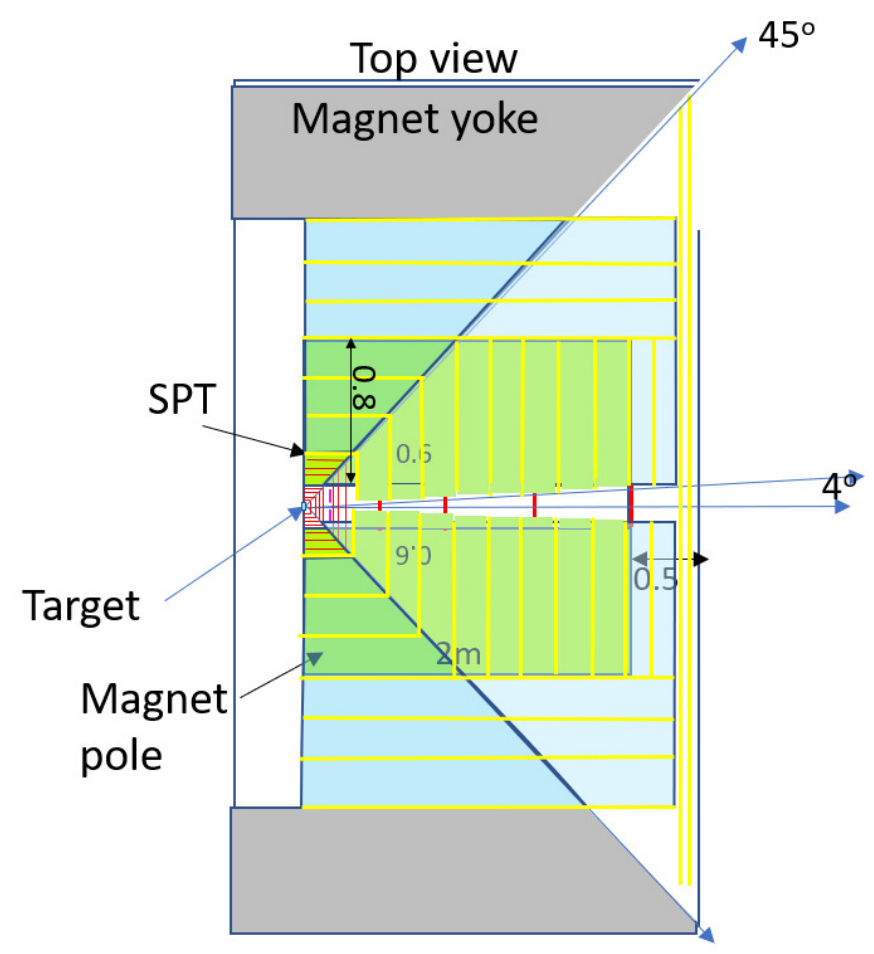

Figure 13: Second stage experimental setup focusing on dilepton (dimuon) measurement.
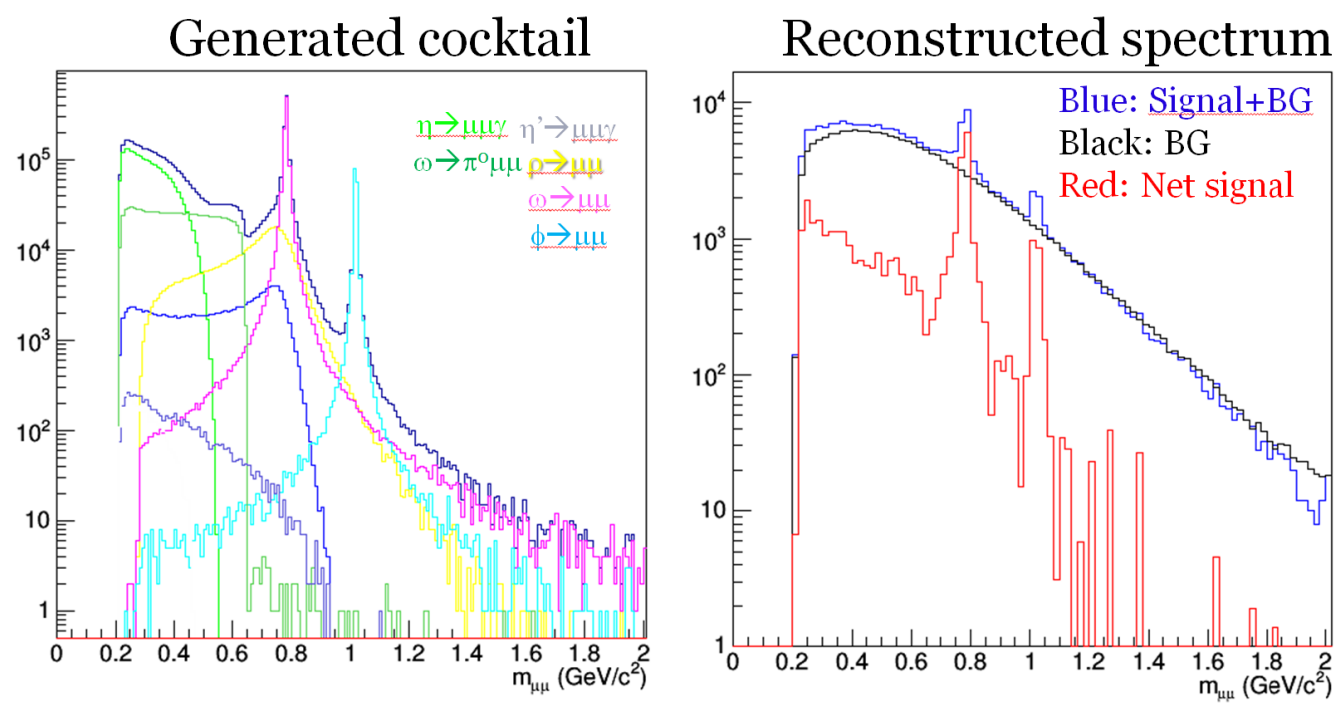

Figure 14: Simulated dimuon mass spectra from various contributions processed through a GEANT detector simulation at J-PARC-HI. (left) Generated dimuon spectra by the modified EXODUS event generator. (right) Reconstructed dimuon spectra from the dimuon signals embedded into JAM events. The branching ratios of hadrons decaying into $\mu^{+} \mu^{-}$are enhanced by a factor of 1000 . 
JAM were decayed into muons by the GEANT4. The collision energy is set to be $\sqrt{s_{N N}}=4.5 \mathrm{GeV}$. The particles passing through the $4 \lambda_{I}$ absorbers were identified as muons. The peaks of $\rho / \omega$ and $\phi$ are visible in the continuous background. Note that in this simulation, the branching ratios of hadrons decaying into $\mu^{+} \mu^{-}$are enhanced by a factor of 1000 in order to earn enough statistics for the signal dimuons.

The third stage experiment focuses on hypernulei measurement. The setup is shown in Fig. 15. The beam will be bombarded to the target followed by the sweeping magnet of around $5 \mathrm{~T}$ and the

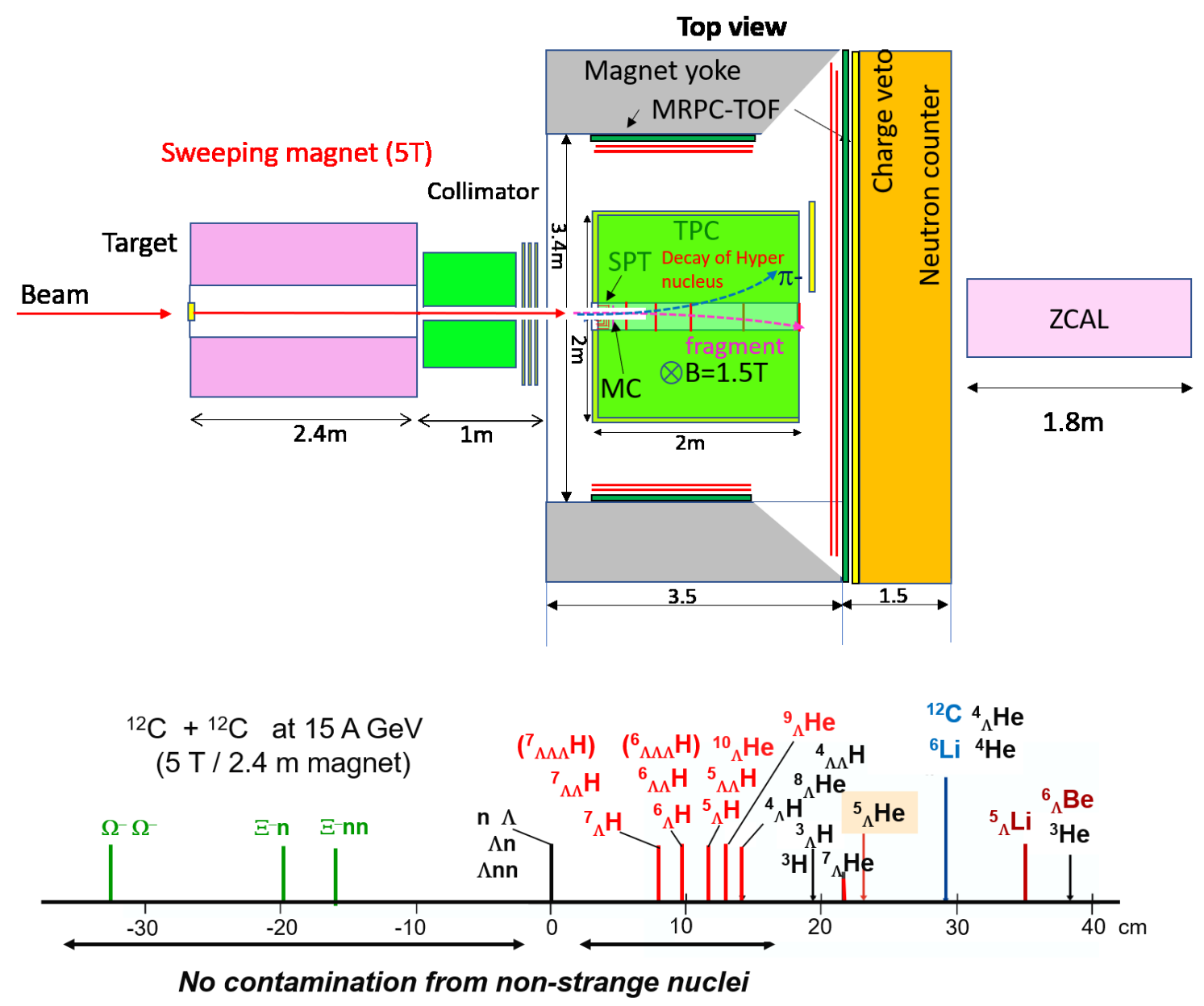

Figure 15: Experimental setup focusing on the hypernuclei (top). The projected position will be different depending on the masses of the hypernuclei (bottom). The ones arriving at the negative position with respect to the beam position will be negatively charged nuclei ever measured.

collimator. The beam particles will be swept away by the magnet and the collimator, and therefore only beam fragments which include hypernuclei will be introduced to the tracking part. The $\pi$ 's coming from the weak decay of the hypernuclei will be tracked and identified by the TPC and the MRPC-TOF, and will be used as tags for hypernuclei events. The projected arrival positions of the hypernuclei at the exit of the collimator will be reconstructed and used for identifying the species of the hypernuclei. The correlation of the projected positions and the species of the hypernuclei is 
shown in the bottom panel of Fig. 15 . This experiment will require very high rate $\left(\sim 10^{8} \mathrm{~Hz}\right)$ since the production cross-section of the hypernuclei is significantly small.

\section{Project status}

The plan of upgrading J-PARC to J-PARC-HI for accelerating heavy ions has been intensively discussed in the Japan Atomic Energy Agency (JAEA) and the J-PARC. The upgrade includes building an ion source, a linac, and a booster to generate and accelerate heavy ions for injecting to the RCS. The cost for the accelerator part has been estimated to be $\sim \$ 150 \mathrm{M}$. We have submitted this proposal for the master plan of science council of Japan, which is the 10-year plan staring from 2020. If successful, the project will be admitted as a prioritized plan and become subject to budget discussion. As for the experimental side, we have identified the location for these detector systems in the J-PARC hadron experimental hall as shown in Fig.16. In the current experimental hall, the

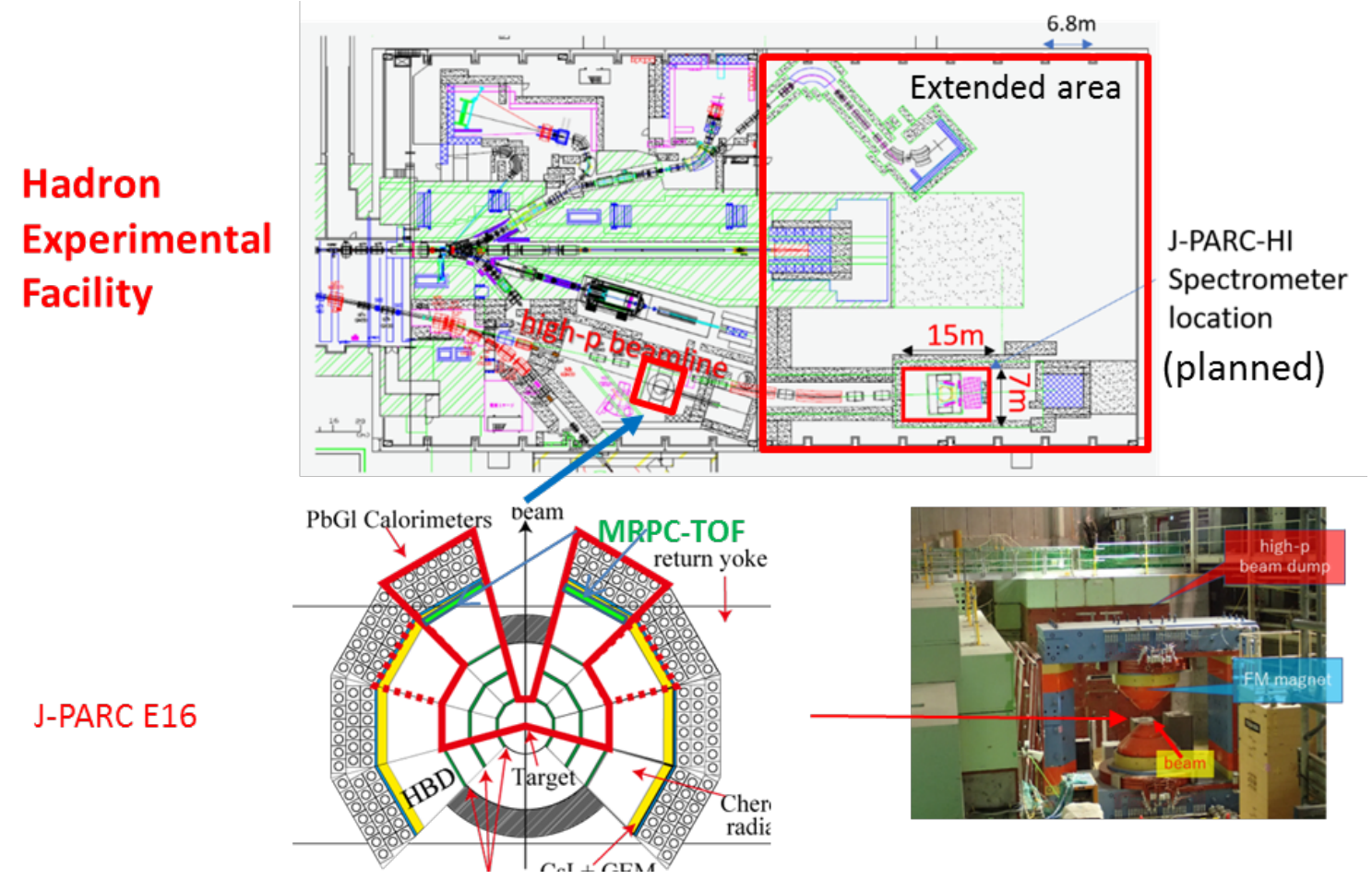

Figure 16: Candidate location for the J-PARC-HI experimental setup. The E16 experiment sitting upstream of the J-PARC-HI beam line is an approved experiment for electron pair measurement, and may be utilized as the day-1 precursor experiment for heavy ion collisions.

primary proton beam coming from the left side of the picture is bombarded to a target and only the secondary particles are transported to experiments. A new beam line called high momentum beam line is being built in the hall to transport the primary beam to experiments. The experimental area is currently limited to the left half of the drawing, but there is a plan to extend the area as shown in the right half of the drawing. The J-PARC-HI will be built on the high momentum beam line in the extended area. On this beam line, there is an approved experiment, E16 [21], being built for lepton-pair measurement using proton-nucleus collisions. We are considering to add time-of-flight 
and centrality detectors to the E16 detector and perform the day-1 precursor experiment with heavy ion beams until the extended area becomes available.

\section{Summary and near term prospect}

In this paper, we discussed the physics perspective of the high density matter study. We introduced the J-PARC-HI project which is a unique lab to tackle the high density matter physics. The world highest rate of heavy ion beam of $10^{11} \mathrm{~Hz}$ is aimed at J-PARC-HI which makes us possible to perform measurements of hadrons, fluctuation of conserved quantities, dileptons, multi-strange hypernuclei. New event selections were also discussed to reach a highest baryon density on the ground. A white paper and a letter of intent were written and submitted in 2016 [22], and the proposal for the master plan of Science Council of Japan was submitted in Mar 2019. Taking the budget situation into account, we anticipate that the day-1 precursor experiment may be performed in 2026 at earliest. The staged detector systems will be built in the extended area of the hadron experimental hall when it becomes available.

\section{References}

[1] FAIR baseline technical report 3a (2006) .

[2] Design and construction of nuclotron-based ion collider facility (NICA) conceptual design report (2008) .

[3] A. Andronic, P. Braun-Munzinger, J. Stachel and H. Stocker, Production of light nuclei, hypernuclei and their antiparticles in relativistic nuclear collisions, Phys.Lett. B697 (2011) 203 [1010.2995].

[4] P. Braun-Munzinger and J. Stachel, Production of strange clusters and strange matter in nucleus-nucleus collisions at the AGS, J. Phys. G21 (1995) L17 [nucl-th/9412035].

[5] BRAHMS collaboration, Nuclear stopping in Au + Au collisions at $\sqrt{s_{N N}}=200$ GeV, Phys. Rev. Lett. 93 (2004) 102301 [nucl-ex/0312023].

[6] Y. Nara, N. Otuka, A. Ohnishi, K. Niita and S. Chiba, Study of relativistic nuclear collisions at AGS energies from $p+$ Be to Au + Au with hadronic cascade model, Phys.Rev. C61 (2000) 024901 [nucl-th/9904059].

[7] A. Andronic, P. Braun-Munzinger and J. Stachel, Hadron production in central nucleus-nucleus collisions at chemical freeze-out, Nucl. Phys. A772 (2006) 167 [nucl-th/0511071].

[8] Y. Akamatsu, M. Asakawa, T. Hirano, M. Kitazawa, K. Morita, K. Murase et al., Dynamically integrated transport approach for heavy-ion collisions at high baryon density, Phys. Rev. C98 (2018) 024909 [1805.09024].

[9] R. Rapp and J. Wambach, Chiral symmetry restoration and dileptons in relativistic heavy ion collisions, Adv. Nucl. Phys. 25 (2000) 1 [hep-ph/9909229].

[10] R. S. Hayano and T. Hatsuda, Hadron properties in the nuclear medium, Rev. Mod. Phys. 82 (2010) 2949 [0 812.1702$].$

[11] M. Kitazawa, T. Koide, T. Kunihiro and Y. Nemoto, Precursor of color superconductivity in hot quark matter, Phys. Rev. D65 (2002) 091504 [nucl-th/ 0111022 ]. 
[12] T. Song, W. Cassing, P. Moreau and E. Bratkovskaya, Open charm and dileptons from relativistic heavy-ion collisions, Phys. Rev. C97 (2018) 064907 [1803.02698].

[13] B. Friman, F. Karsch, K. Redlich and V. Skokov, Fluctuations as probe of the QCD phase transition and freeze-out in heavy ion collisions at LHC and RHIC, Eur. Phys. J. C71 (2011) 1694 [1103.3511].

[14] Y. Nara, H. Niemi, J. Steinheimer and H. Stöcker, Equation of state dependence of directed flow in a microscopic transport model, Phys. Lett. B769 (2017) 543 [1611. 08023 ].

[15] L. Schachinger, G. Bunce, P. Cox, T. Devlin, J. Dworkin et al., A Precise Measurement of the $\Lambda^{0}$ Magnetic Moment, Phys.Rev.Lett. 41 (1978) 1348.

[16] C. Ankenbrandt, J. Berge, A. Brenner, J. Butler, K. Doroba et al., A Precise Measurement of the Sigma+ Magnetic Moment, Phys.Rev.Lett. 51 (1983) 863.

[17] STAR collaboration, $\Lambda \Lambda$ Correlation Function in Au+Au collisions at $\sqrt{s_{N N}}=200$ GeV, Phys. Rev. Lett. 114 (2015) 022301 [1408.4360].

[18] S. Gongyo et al., Most Strange Dibaryon from Lattice QCD, Phys. Rev. Lett. 120 (2018) 212001 [1709.00654].

[19] PHENIX collaboration, Detailed measurement of the $e^{+} e^{-}$pair continuum in $p+p$ and Au+Au collisions at $\sqrt{s_{N N}}=200 \mathrm{GeV}$ and implications for direct photon production, Phys. Rev. C81 (2010) 034911 [0912.0244].

[20] GEANT4 collaboration, GEANT4: A Simulation toolkit, Nucl. Instrum. Meth. A506 (2003) 250.

[21] S. Yokkaichi et al.Proposal for J-PARC E16 (2006) .

[22] http://asrc.jaea.go.jp/soshiki/hadron/jparc-hi . 